\title{
SYSTEMATIC GENERATION OF A 3D DSM BY EXTRACTING SOCIAL ROBOT BEHAVIORS FROM LITERATURE
}

\author{
Ozer, Ilayda; Erden, Zuhal
}

Atilim University

\begin{abstract}
Social robots are in direct communication and interaction with people, thus it is important to design these robots for different needs of individuals or small groups. This has revealed the need to develop design methods for personalized or mass-individualized social robots, which are expected to respond to many different needs of people today and in the future. In this paper, a previously developed 3D DSM model is implemented in the systematic conceptual design of social robot families. The model is independent of any physical elements and based on behavioural elements as perception, cognition and motoric action. The data regarding 45 different social robots from 80 articles in the literature is used to identify these three behaviours of the existing social robots and the mutual relationships among these different behaviours are defined in order to develop a 3D DSM structure to be used as a basis for designing social robot families. The resulting novel 3D DSM is a general-purpose, basic model that can be used to identify behavioural modules to design social robot families.
\end{abstract}

Keywords: Design methods, Product families, Conceptual design, Mechatronics

\section{Contact:}

Erden, Zuhal

Atilim University

Atilim University

Turkey

zuhal.erden@atilim.edu.tr 


\section{INTRODUCTION}

It has been a technological anticipation that robots will become widespread and find many application areas in almost all sectors such as defence, transportation, industry, manufacturing, agriculture, health/elderly care as well as in medicine, industrial automation, and service systems. This process continues to expand, and the role of robots in our daily life continues to increase. With the technological development and spread of robots, human-robot relations are also expanding as social robots are increasingly equipped with features to provide human-robot interaction and, hence, gain more importance in our lives. Various social and individual needs in public areas such as shopping centres, museums, government buildings, hospitals, parks, etc. could be fulfilled by social robots in this way. These are autonomous agents capable of communicating and interacting with people in behaviour and emotional dimensions (Campa, 2016). Currently, almost 30 million robots, including service and industrial robots, are active in the world and $94 \%$ of them are service robots. Of these, $15 \%$ engage in social interactions (KPMG Advisory N.V., 2016). Aibo, Asimo, Furby, Nao are famous examples of robots with social skills.

With increasing development of social robots and their use in many different areas, the need for these robots in other fields continues to increase and diversify. Since, by definition, social robots are in direct communication and interaction with people, it is important that they are designed for different needs of individuals or small groups. This has revealed the need to develop design methods for personalized or mass-individualized social robots, which are expected to respond to many different needs of people today and in the future. As a result, they will need to be designed as custom-fit almost entirely in the long term. Therefore, systematic design methods came into the picture. In design science, product customization is achieved via platform-based modular design approach, which results in the development of product families. These refer to a set of similar products that are derived from a common platform and, yet, possess specific features/functionalities to meet particular customer requirements (Jiao et al., 2007) by assembling/integrating different modules. This design approach can also be used to customize social robots by developing a systematic method suitable for such systems. In the modular design literature, there are three systematic methods for product modularization as the heuristic method (Stone et al., 2000), modular function deployment (MFD) (Erixon, 1998), and design structure matrix (DSM) (Pimmler and Eppinger, 1994; Browning, 2001). Heuristic method is based on the functional modelling approach used in systematic design (Pahl and Beitz, 1996) for identifying modules from the function structure of a product. This method is applicable to all kinds of products, and it is independent of any physical realization. However, in this method, the functional structure of the product is designer-dependent, resulting in the problem of changing modules with changing designers, or even as a function structure is changed by a single designer. This situation weakens the objectivity expected from any systematic modular design methodology. Later, a systematic methodology was proposed to create a product portfolio by extending the heuristic method (Dahmus et al., 2001). However, in that study, modularization is made at an advanced stage (preliminary design stage) of the conceptual design after determining the physical principles for the product portfolio. What's more, it does not consider modularity decisions needed to be done at a higher level of abstraction, where no physical principle is associated with the required functions and/or behaviours. Therefore, the method is not suitable for developing mechatronic product families. Since the related philosophy requires both an interdisciplinary and a multidisciplinary approach, for which modularity decisions should are made at the upstream design stage. Modular function deployment (MFD) is also based on functional decomposition similar to heuristic method. However, it is developed for the modularization of a product over time (Erixon, 1998). MFD is considered as a management-oriented method (Höltta and Salonen, 2003) because, besides functionality, it takes other factors affecting modularity into account. MFD also disregards the interfaces between the functions of the product and rather focuses on the benefits of more strategic issues, such as modularity, ease of maintenance and recycling, which are not thought much of by other methods. In the design structure matrix (DSM) method (Pimmler and Eppinger, 1994; Browning, 2001), the functional or physical elements of a product are placed in the rows and columns of a 2D matrix structure. Energy, material, information and/or spatial interactions between elements/functions are determined. Then, the elements/functions are clustered into modules in such a way that interactions within the same module are maximum and those across different modules are minimum. Since this type of clustering and modularization are reproducible and verifiable, DSM is a 
more objective and engineering-oriented method. DSM is mainly implemented during the later stages of the conceptual design where the physical components of a system are determined.

The above-mentioned systematic modular design methods to develop product families are mainly concentrated on mechanical systems. In addition, the methods used in the modular design of mechanical systems in the literature are those related to the physical aspects of the system; as such, in order to use these methods, the system must emerge as a physical design. The modular structure of robots, however, is based on mechatronic design philosophy that requires focusing on the function and behaviour of the system from the early stage conceptual design phase. Consequently, a need arises for a general systematic structure which to be used at the early conceptual design of modular robots, independent of physical elements, based on functional/behavioural elements and applicable to different robots. As a contribution to developing a systematic method for robot family designs, a conceptual framework was developed by using a behaviour-based robot design approach (Araz and Erden, 2014). The conceptual framework suggests a 3D DSM structure (Erden, 2018), which is developed entirely based on the 2D DSM approach in the literature, can be used systematically in the early conceptual design stage of the platform-based modular robots with various case studies and laboratory applications (Ayhan and Erden, 2016, Erden, 2017, Ozer and Erden, 2018).

The 3D DSM model is developed for a systematic conceptual design of robot families; therefore, it is independent of any physical elements and uses behavioural elements as perception, cognition and motoric action. In order to implement this model in the systematic conceptual design of social robot families, it is necessary to identify the behavioural elements in the three groups mentioned above and to define the relationships between these elements. In the present paper, the authors make use of the data from the literature to identify the perception, cognition and motoric action behaviours of existing social robots and define the mutual relationships among these different behaviours in order to develop a 3D DSM structure to be used as a basis for designing social robot families. In all, 45 different social robots from 80 articles were examined. The resulting novel 3D DSM is a general-purpose, basic model that can be used to identify behavioural modules to design social robot families.

The remaining part of this paper is organized as follows: Section 2 gives a brief description of the previously developed conceptual framework for the 3D DSM structure to develop robot families based on robot behaviours. Section 3 provides a review of the social robot literature and explains the related behavioural elements for social robots as well as the construction of 3D DSM using the relationships among these elements. Finally, in Section 4, discussion and conclusions regarding this work are provided with future research directions.

\section{3D DSM APPROACH FOR SOCIAL ROBOT FAMILY DESIGN}

In order to facilitate a systematic design for platform-based social robot families for mass customization, we use a novel 3D DSM approach, based on three types of mechatronic behaviour modules as perception, cognition and motoric action. The conceptual infrastructure of 3D DSM uses a behavioural design approach. In accordance to the general characteristics of a mechatronic system's operational behaviour at the highest level of abstraction, composed of three states as perception, cognition and motoric action represented as an event-driven (Discrete-Event System Specification-DEVS) model (Araz and Erden, 2014). The system communicates its environment to collect and process data during the perception state. In the cognition state, the data is used with proper reasoning and decision-making to respond to changes in the environment. In the motoric action state, task execution is performed physically in accordance with decision-making and/or as a reflexive response to the changes in the environment. Perception is decided as the initial state, because once the system starts its operation, it is expected to collect data from the environment for processing and decision-making to create a motoric action. The environment outside a mechatronic system is defined as a physical medium, which includes the physical world and other mechatronic/non-mechatronic systems. An important characteristic of the behavioural design approach is that the behavioural model is independent of physical subsystems and/or components. Thus, designers are allowed to consider various physical system alternatives to accomplish the required behaviours, while elaborating the design at lower levels of abstraction. This characteristic is crucial for the design of mechatronic systems and robots since these systems are differentiated by synergistic integration that can be achieved by domain independence.

This domain-independent behavioural design approach is used to develop an initial conceptual scheme for the modular design of robots (Erden, 2018). For this purpose, a novel 3D DSM approach is 
developed based on the Design Structure Matrix (DSM), which is an important representation and analysis tool for system modelling, particularly for decomposition and integration purposes (Browning, 2001). A DSM is a matrix representation of a system, in which the components are listed in the first row and the first column (Li et al., 2013). Off-diagonal cells indicate the interactions (i.e., dependency, information flow, etc.) among the system elements. Various researchers have worked on DSM for modularization and clustering (Helmer et al., 2010; Li and Xie, 2015; Qiao et al., 2017). In these studies, the system components are assumed to be known, such that the interactions could be identified. Using clustering techniques, modularization on an existing system could be successfully performed using this 2-dimensional matrix structure.

In this study, mechatronic behaviour modules are represented using a 3-dimensional matrix (array) structure, called 3D DSM, and adopted from the classical 2D DSM approach. Figure 1 represents the structure of 3D DSM, in which modules for perception (P), cognition (C) and motoric action (M) behaviours are placed on mutually perpendicular axes. Figure 1 illustrates that there exist three mechatronic products in the family and as an example they are treated as three robots denoted by 3 different labels as a square for Robot 1 (R1), a triangle for Robot 2 (R2) and a circle for Robot 3 (R3). The relationships among these three types of modules are determined based on the characteristics of the intended operational behaviour of the mechatronic products (robots are considered as an example) in the family. For example, one can consider perception module P1. If the output information of P1 is processed by the cognition module $\mathrm{C} 1$ (a relationship between $\mathrm{P} 1$ and $\mathrm{C} 1$ ) resulting in a motoric action M1 (a relationship between $\mathrm{C} 1$ and M1), this will form a mutual relationship between P1, C1 and M1 represented with a cubic element in the corresponding cell in the 3D DSM.

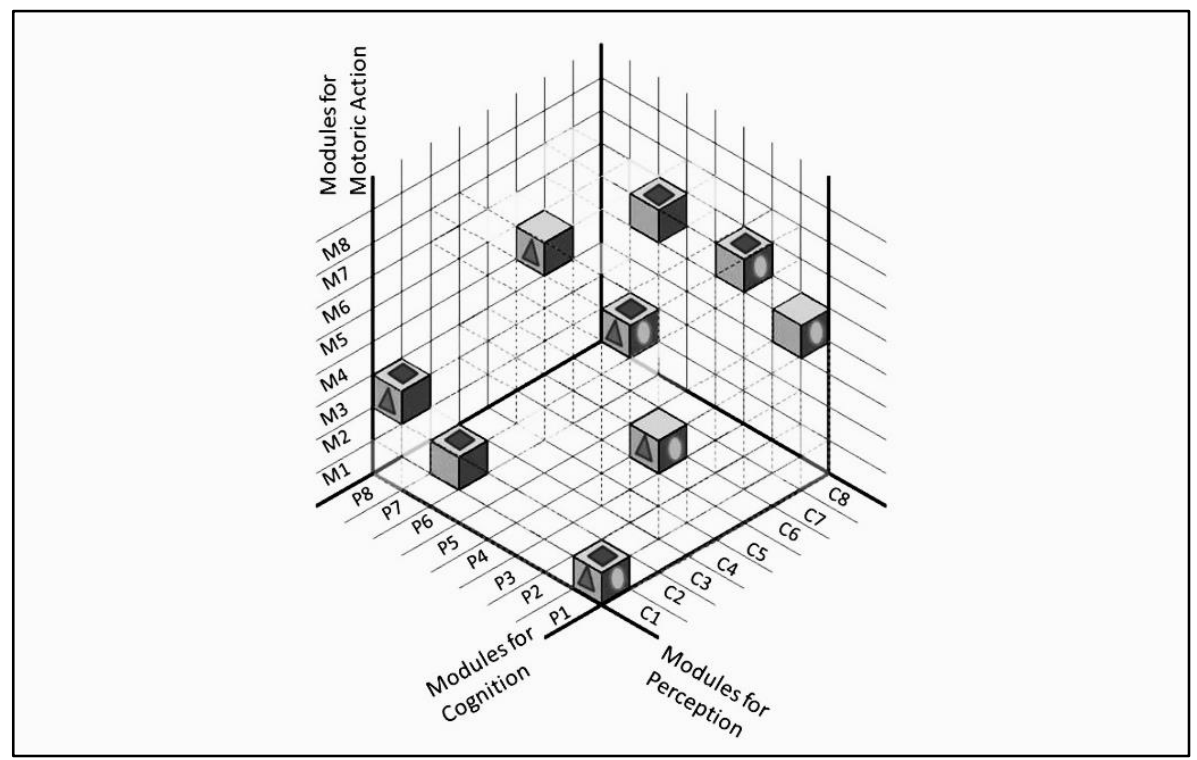

Figure 1. Representation of mechatronic behaviour modules using 3D DSM (Erden, 2018)

It can be noticed from Figure 1 that the P1-C1-M1 relationship exists in all three robots in the family. In another case, if the output information of $\mathrm{P} 1$ is processed by the cognition module $\mathrm{C} 8$ resulting in a motoric action M5, this will form a mutual relationship between P1, C8 and M5. This combination exists only in the R3's behaviour as illustrated in Figure 1. Using different mutual relationships among perception, cognition and motoric action modules, one can design various robots in a robot family in terms of their behaviours. By using 3D DSM, symbolic representations of mechatronic behaviour modules are generated and used in describing robot tasks. The 3D DSM approach constructs a framework to support a systematic platform-based mechatronic design rather than precisely define mechatronic behaviour modules, hence the use of simplifications are required. For example, "perception behaviour modules" are considered directly as sensors in a physical realization, and we describe perception modules symbolically using well-known principles of related sensors which are frequently used in mechatronic systems. "Cognitive behaviour modules" are described as software modules, which process the output data provided by the perception behaviour modules and make a decision on an appropriate motoric action, resulting in the execution of tasks for the intended robot behaviour. 


\section{SOCIAL ROBOT BEHAVIOURS AND THEIR INTERACTIONS}

In the present study, it is aimed to identify social robot behaviours and their relationships using data from the literature in order to generate a 3D DSM structure. For this purpose, a survey of social robots is carried out, resulting in the examination of 45 different robots from 80 articles. The information about the functions and behavioural characteristics of these robots is collected and, then, the behavioural characteristics of the robots are classified according to the perception, cognition and motoric action behaviours of the 3D DSM infrastructure. In the selection of the social robots examined in this study, the diversity of the tasks taken up by these robots in different areas is considered. Each of the examined social robots has unique behaviours that change according to their usage areas. Some of these robots behave as companions at home and help people in their daily routine work, such as NAO (Cao et al., 2018), Travis (Birnbaum et al., 2016), Zenbo (Chen et al., 2018), Jibo (Guizzo, 2016), Robovie (Kahn et al., 2008) domestic robots (Xiong et al., 2009), Maggie (Salichs et al., 2006), a small window-cleaning robot (Liu et al., 2009), Buddy (Potnuru et al., 2016), Reem-B (Téllez et al., 2008) and ASIMO (Okita et al., 2009). Some of them look like toys and tell stories, recognize other people, communicate and play games. Aibo, iCat, Barthoc (Hegel et al., 2007), Lino (Salichs et al., 2006) and Tega (Kory Westlund et al., 2016) are examples of such robots. Valerie (Gockley et al., 2005), Pearl (Broekens et al., 2009), fire-fighting robot (Rangan et al., 2013) and Robotman (Lopez et al., 2017) are service robots, which perform various tasks mainly at a workplace. Another important usage area of social robots is patient-companion; Huggable (Stiehl et al., 2006), CPAC, Bobus (Alhaddad et al., 2018), Paro (Broekens et al., 2009) and Pepper (Cao et al., 2018) are examples of this kind of robots. Social robots are also used for education (Ferguson et al., 2011; Gockley et al., 2005) and communication (Lee and Yoo, 2017; Zaraki et al., 2014; Aryananda, 2002; Ađalgeirsson and Breazeal, 2010).

The behavioural characteristics of 45 robots in the literature are determined and 26 perception behaviours, 45 cognition behaviours and 58 motoric action behaviours are identified and defined, as in the 3D DSM model. As an example, Table 1 depicts some of these behaviours for 5 robots selected among the 45 robots.

Table 1. Example robot behaviours for perception, cognition and motoric action

\begin{tabular}{|l|l|l|l|}
\hline Perception & Cognition & Motoric Action & $\begin{array}{l}\text { Social robot examples } \\
\text { in literature }\end{array}$ \\
\hline Detect fire & $\begin{array}{l}\text { Decision on the existence } \\
\text { of fire }\end{array}$ & Fire extinguishing & Fire Fighting Robot \\
\hline Detect danger & $\begin{array}{l}\text { Decision for going to } \\
\text { where the sound is }\end{array}$ & $\begin{array}{l}\text { Going to where the } \\
\text { sound is }\end{array}$ & NAO \\
\hline $\begin{array}{l}\text { Detect a } \\
\text { shopping } \\
\text { command }\end{array}$ & $\begin{array}{l}\text { Decision for scanning } \\
\text { where the sound is }\end{array}$ & $\begin{array}{l}\text { Scanning where the } \\
\text { sound is }\end{array}$ & Robotman, Robovie, Jibo \\
\hline Detect touch & $\begin{array}{l}\text { Decision for connecting } \\
\text { to the Internet for } \\
\text { shopping }\end{array}$ & Ordering & Robotman, Zenbo \\
\hline
\end{tabular}

In the next stage of the study, the interactions among the perception, cognition and motoric action behaviours of the robots detected from the literature are determined using a systematic method based on the 2D DSM structure (Helmer et al., 2010). The interactions among different types of robot behaviours during the early stages of the conceptual design are mainly triggered by information transfer or data exchange. Therefore, we consider these interactions in terms of information flow between both 'perception-cognition' and 'cognition-motoric action' separately and, then, represent all of these interactions in a 3D structure as depicted in Figure 2.

Firstly, the information flow between the "perception-cognition" behaviours for social robots examined in the literature is classified into 4 groups as shown in Figure 2(a). 'I_audio' shows an audio flow of information. For example, when a voice command is sent to a social robot, this command is detected by the robot. 'I_image' indicates that the information is transmitted as an image. As an example, for this kind of interaction, a social robot understands the existence of an object by detecting its image. 'I_touch' refers to the state of information transfer by touch or heat. For example, an 
entertainment robot may be designed such that it decides to sing when somebody touches it. 'I_smell' represents that the interaction is via smell. For example, a guard robot can detect the smell of gas and decide, using this information, what to do. A rating scheme is used for these four groups of information flows based on the social robot behaviours examined in the literature. According to the scheme, the value " 0 " indicates that there is no interaction and " 1 " means that an interaction exists, but it is not determinative for cognition behaviour. A value of " 2 ", however, indicates a dominant interaction between "perception" and "cognition" behaviours. The example Figure 2 (a) represents the interaction between "detect an object"(a "perception" behaviour) and "decide to grab the object" (a "cognition" behaviour) based on the above-mentioned rating scheme. In order for the object to be recognized, the robot must first collect information from the environment. Receiving voice information varies depending on whether or not the object produces sounds. Therefore, the value of 'I_audio' is " 1 ". More important than voice information, the image of the object is used for recognition. Therefore, 'I_image' is given a value of "2". However, it is assumed that the robot does not need interaction via touching or smell for detecting an object. Thus, values for 'I_touch' and 'I_smell' are both "0". This type of interaction exists in NAO robot (Cao et al., 2018).

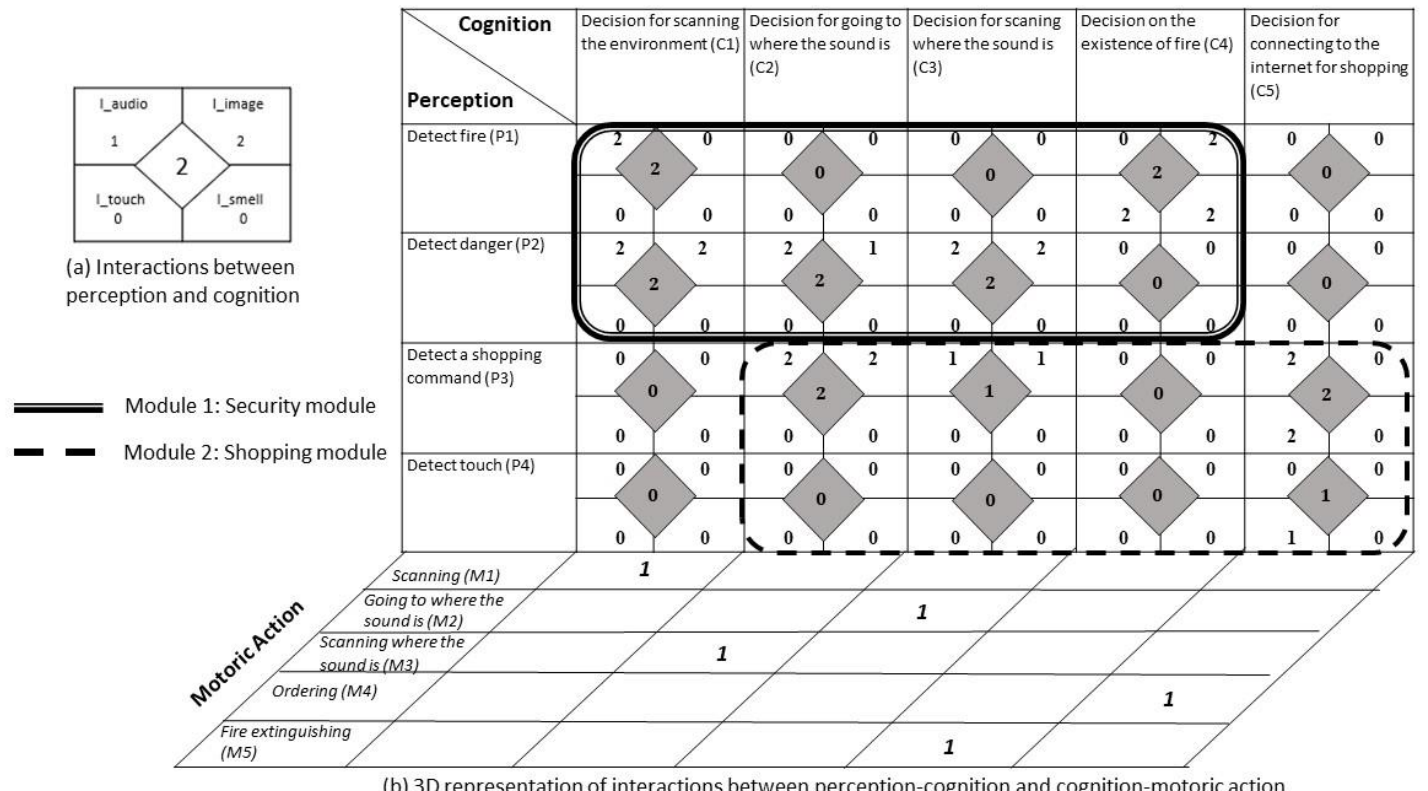

Figure 2. Interactions between perception, cognition and motoric action

The above-mentioned interactions between perception and cognition are extracted for all the robots studied in the literature and are shown as a general systematic formal structure as an "mxn" matrix (PC). Interactions between cognition and motoric action behaviours are defined as either "interaction exists" or "interaction does not exist" and they are represented by an "nxk" matrix (CM). If an element in the CM matrix represents an interaction, then its value is " 1 "; otherwise, it is " 0 ". The two matrices, $\mathrm{PC}$ and $\mathrm{CM}$, are given in Equation (1):

$P C=\left[\begin{array}{ccc}P C_{11} & \cdots & P C_{1 n} \\ \vdots & \ddots & \vdots \\ P C_{m 1} & \cdots & P C_{m n}\end{array}\right] \quad C M=\left[\begin{array}{ccc}C M_{11} & \cdots & C M_{1 k} \\ \vdots & \ddots & \vdots \\ C M_{n 1} & \cdots & C M_{n k}\end{array}\right]$

For the 45 social robots examined in the literature, the PC and CM matrices are defined separately as 2D DSMs and, then, combined as a 3D structure. Figure 2(b) illustrates an example 3D representation for the interactions among perception, cognition and motoric action behaviours including those given in Table 1. The resulting PC and CM matrices are, then, combined to show the interactions among 3 different behavioural elements, and the 3D DSM structure is obtained as a result.

Examining the interactions between perception, cognition, and motoric action behaviours of 45 social robots in the literature led to the identification of 11 'mechatronic behaviour modules'. Two of them are schematically represented in Figure 2(b). The security module detects and extinguishes the fire in the robot's environment. This module also detects the sound that is a sign of danger and scans the 
environment. The shopping module detects a voice command for shopping and also detects touch and, then, performs shopping by connecting to the Internet. In this study, the mechatronic behaviour modules were determined by using the systematic approach developed for 3D DSM based on the relationships between perception, cognition and motoric action behaviours of social robots in the literature. For example, consider the security module in Figure 2(b): a robot providing security must scan the environment where it is located to detect a fire or any danger. Such a robot also makes a sound scan to understand if there is an undefined sound in the environment. After detecting the sound, it will also scan the environment to determine the location where the sound is. On the other hand, if the robot detects fire, it will put it out. The 'perception-cognition' interactions necessary for these behaviours of the robot were determined and represented as a 2D DSM (PC matrix) as shown in the upper-left rectangular region in Figure 2(b). Then, using cross-interactions with another 2D DSM (CM matrix) formed by 'cognition-motoric action' relationships, a 3D DSM structure resulting in the 'security module' is constructed. Using a similar logic, the 'shopping module' is created as a second module. Figure 3 graphically shows the resulting 3D DSM structure, where the small elements indicate the security module and the big elements show the shopping module.

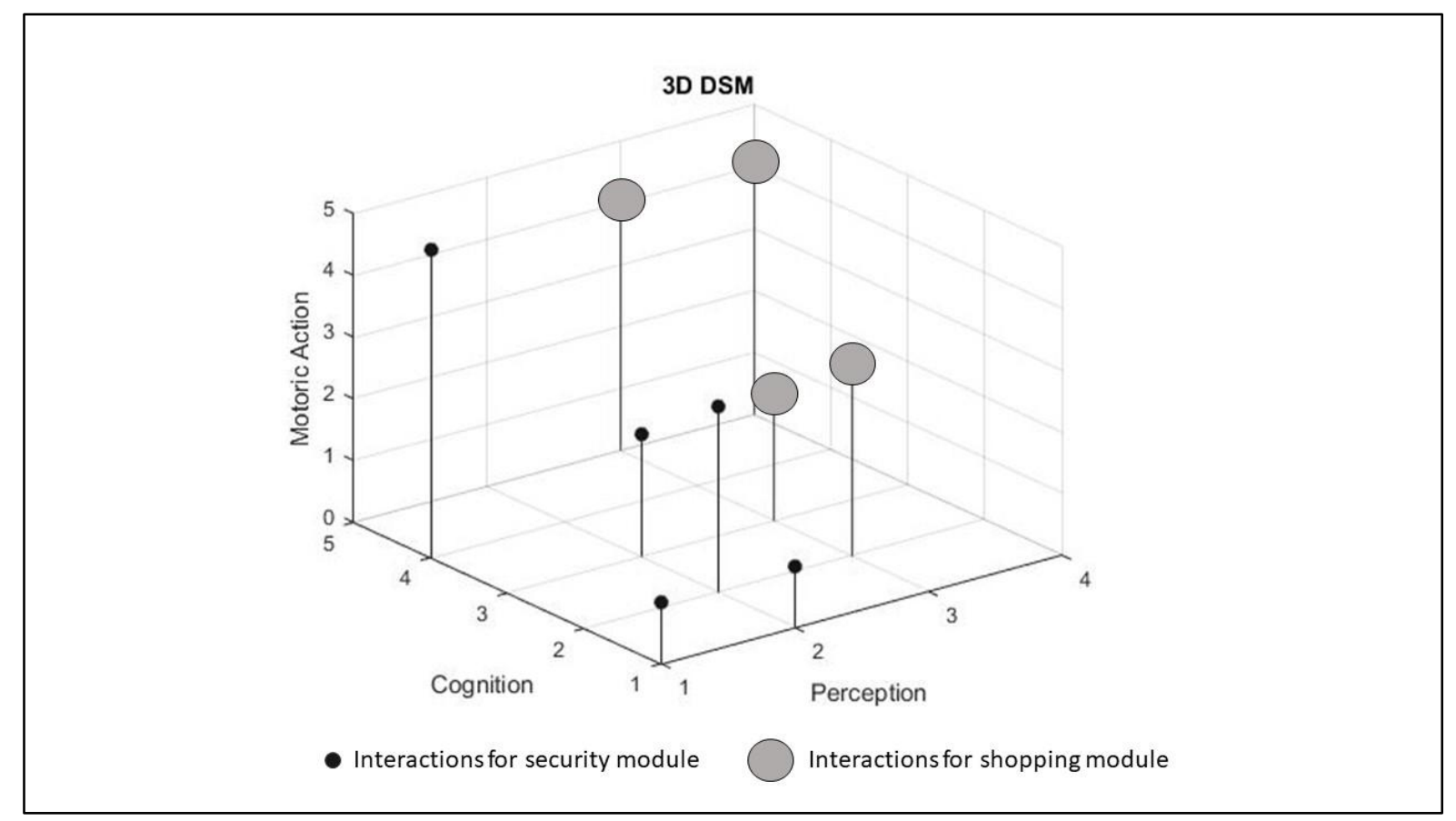

Figure 3. 3D DSM for the interactions given in Figure 2(b)

For the 45 social robots examined in this study, 11 different behaviour modules were defined by using the systematic method described in the example of the security module. The robots studied consist of different combinations of these modules. Figure 4 shows the module combinations for some of these robots as a conceptual structure, which allows the new robots to be designed in such a way so as to perform different tasks by using different combinations of modules. For example, Robot 6 in Figure 4 symbolizes a new robot that can be designed using a different combination of the existing modules to guide a disabled person. By using this methodology and the conceptual structure, it is possible to add personalized robots to the current social robot family and meet, in this way, the different needs of the society. In doing so, designers can use different combinations of the existing modules, or they can work to add new 'mechatronic behaviour modules' to the module repository. Thus, in the early stages of the conceptual design, personalized and/or mass-customized social robots that meet different social needs can be developed systematically. 


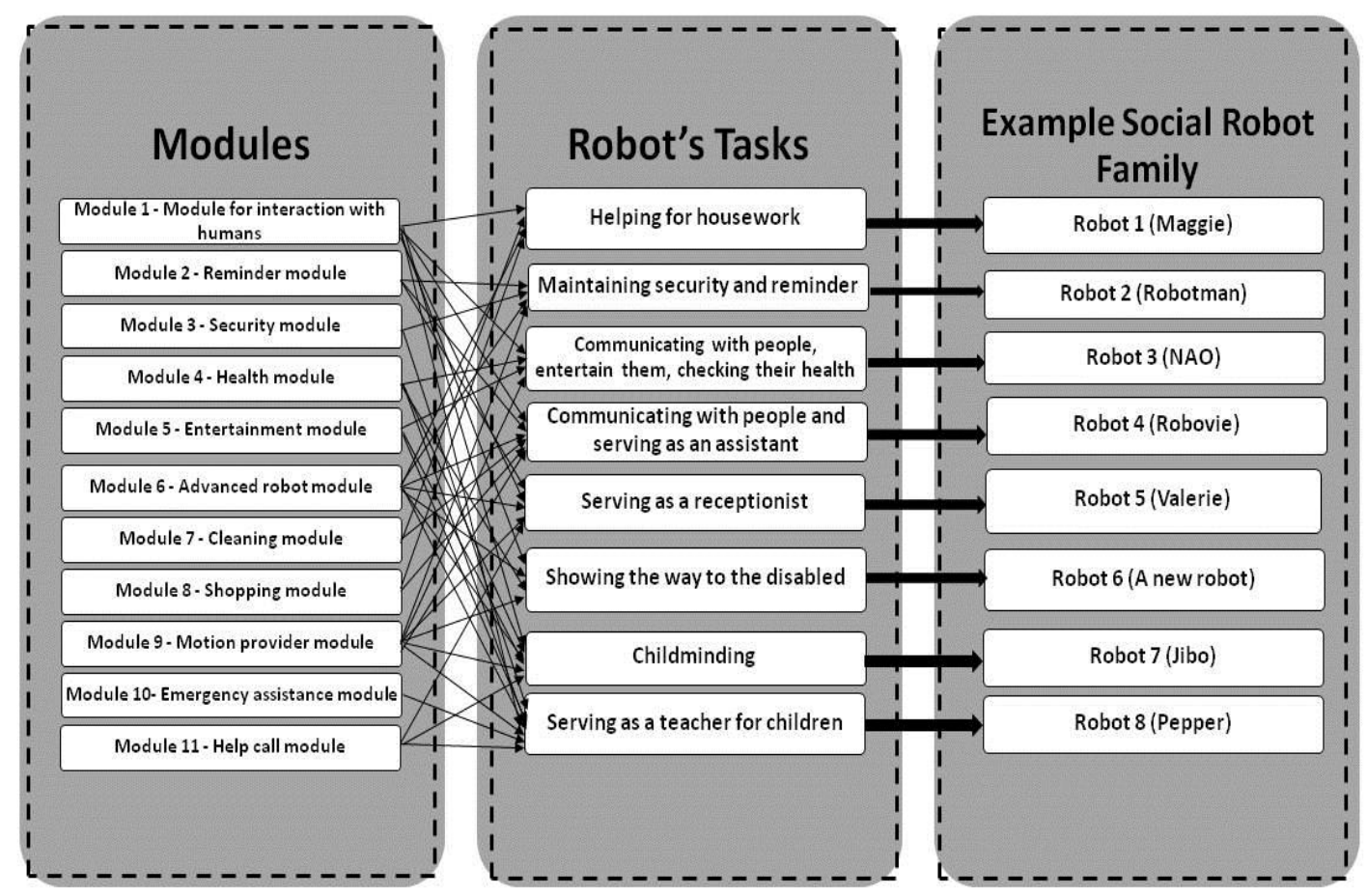

Figure 4. Conceptual structure for a social robot family with behavioural modules

\section{DISCUSSION AND FUTURE WORK}

In addition to the robots used in industrial or special applications, social robots have gained importance in recent years and are increasingly used to meet many different needs and perform various tasks in the daily lives of ordinary people. This variety of tasks requires a systematic design of social robots in the form of product families. In this article, a 3D DSM infrastructure is developed which can be used in the systematic conceptual design of the social robot families by extracting the data obtained from the literature. In this study, a total of already existing 45 social robots are investigated to determine their basic perception, cognition and motoric action behaviours. Then, the interactions among these robot behaviours are defined and the corresponding 3D DSM is constructed. The 3D DSM is obtained mainly by using a 2D DSM methodology, such that two-sided interactions between 'perception-cognition' (PC) and 'cognition-motoric action' (CM) behaviours of the social robots are identified separately. Then, using cross combinations, mutual interactions among these three types of behaviours are generated resulting in a novel 3D DSM structure. It should be emphasized that, although we have been inspired by the 2D DSM structure existing in the literature to represent the above-mentioned two-sided interactions, our approach offers an important contribution. The two 2D DSMs in the present study, namely PC and CM, are rectangular matrices that combine different types of elements. Thus, the 3D DSM structure generated in this way appears as a three dimensional array with different type and number of elements in each dimension. The present study formulates a conceptual framework for mass-customized social robot design methodology by elaborating on the systematic generation of a 3D DSM structure that could be used for module identification. All in all, this study forms a basis to contribute to the development of a general systematic method for the design of social robot families. Within the scope of this study, systematic module identification was performed manually; however, future research will be focused on the clustering of triple interactions and automatic generation of 'mechatronic behaviour modules' for social robots.

\section{REFERENCES}

Adalgeirsson, S.O. and Breazeal, C. (2010), "MeBot: a robotic platform for socially embodied presence", The 5th ACM/IEEE International Conference on Human-Robot Interaction, Osaka, 2-5 March 2010, IEEE

Press, Piscataway, NJ, USA, pp. 15-22. 
Alhaddad, A.Y., Javed, H., Connor, O., Banire, B., Al Thani, D. and Cabibihan, J.J. (2019), "Robotic Trains as an Educational and Therapeutic Tool for Autism Spectrum Disorder Intervention", International Conference on Robotics and Education, Malta, 18-20 April 2018, Advances in Intelligent Systems and Computing, Vol 829. Springer, Cham, pp. 249-262.

Aryananda, L. (2002), "Recognizing and remembering individuals: Online and unsupervised face recognition for humanoid robot”, IEEE/RSJ International Conference on Intelligent Robots and Systems, Lausanne, 30 Sept. - 4 Oct. 2002, IEEE, Piscataway, NJ, USA, pp. 1202-1207. http://doi.org/10.1109/IRDS.2002.1043897.

Araz, M. and Erden, Z. (2014), "Behavioural representation and simulation of design concepts for systematic conceptual design of mechatronic systems using Petri Nets", International Journal of Production Research, Vol. 52 No. 2, pp. 563-583. https://doi.org/10.1080/00207543.2013.838648.

Ayhan, E. and Erden, Z. (2016), "Implementation of Function Structure Heuristics for Modular Design of an Educational Mechatronic Product Family”, 17th International Conference on Machine Design and Production (UMTIK 2016), Turkey, 12-15 July 2016, Bursa.

Birnbaum, G.E., Mizrahi, M., Hoffman, G., Reis, H.T., Finkel, E.J. and Sass, O. (2016), "What robots can teach us about intimacy: The reassuring effects of robot responsiveness to human disclosure"“, Computers in Human Behaviour, Vol. 63, pp. 416-423. https://doi.org/10.1016/j.chb.2016.05.064.

Broekens, J., Heerink, M. and Rosendal, H. (2009), “Assistive social robots in elderly care: a review”, Gerontechnology, Vol. 8 No. 2, pp. 94-103.

Browning, T.R. (2001), "Applying the design structure matrix to system decomposition and integration problems: a review and new directions", IEEE Transactions on Engineering Management, Vol. 48 No. 3, pp. 292-306.

Campa, R. (2016), "The rise of social robots: a review of the recent literature", Journal of Evolution and Technology, Vol. 26 No. 1, pp. 106-113.

Cao, H.L., Van de Perre, G., Kennedy, J., Senft, E., Esteban, P.G., De Beir, A., Simut, R., Belpaeme, T., Lefeber, D. and Vanderborght, B. (2018), "A personalized and platform-independent behaviour control system for social robots in therapy: development and applications", IEEE Transactions on Cognitive and Developmental Systems, http://doi.org/10.1109/TCDS.2018.2795343.

Chen, G.D., Hsu, T.C. and Liyanawatta, M. (2018), "Designing and implementing a robot in a digital theatre for audience involved drama-based learning", International Conference on Innovative Technologies and Learning, Portoroz, Slovenia, August 27-30, 2018, Springer, Cham, LNCS Series Vol. 11003, pp. $122-131$.

Dahmus, J.B., Gonzalez-Zugasti, J.P. and Otto, K.N. (2001), "Modular product architecture”, Design Studies, Vol. 22 No. 5, pp. 409-424. https://doi.org/10.1016/S0142-694X(01)00004-7.

Erden, Z. (2017), "Smazzle: A Demonstrative Case Study for Modular Design of Mechatronic Products", International Conference on Engineering Technologies, Konya, Turkey, December 7-9.

Erden, Z. (2018), "Development and Implementation of Behavioural Modules for Platform-Based Mechatronic Design", The 12th Int. Symp. on Tools and Methods of Competitive Engineering (TMCE 2018), Spain, May 7-11, 2018, Las Palmas de Gran Canaria, pp. 625-634.

Erixon, G. (1998), Modular function deployment: a method for product modularisation, Royal Institute of Technology, Department of Manufacturing Systems, Assembly Systems Division, Stockholm.

Ferguson, M., Webb, N. and Strzalkowski, T. (2011), "Nelson: a low-cost social robot for research and education", The 42nd ACM Tech. Symp. on Computer Science Education, Dallas, TX, USA, March 9-12, 2011, ACM, pp. 225-230.

Gockley, R., Bruce, A., Forlizzi, J., Michalowski, M., Mundell, A., Rosenthal, S., Sellner, B., Simmons, R., Snipes, K., Schultz, A.C. and Wang, J. (2005), “Designing robots for long-term social interaction”, IEEE/RSJ International Conference on Intelligent Robots and Systems, Edmonton, Canada, August 2-6, 2005, IEEE, pp. 1338-1343. http://doi.org/10.1109/IROS.2005.1545303.

Guizzo, E. (2016), “The little robot that could... Maybe”, IEEE Spectrum, Vol. 53 No. 1, pp. 58-62. http://doi.org/10.1109/ MSPEC.2016.7367471.

Hegel, F., Lohse, M., Swadzba, A., Wachsmuth, S., Rohlfing, K. and Wrede, B. (2007), "Classes of applications for social robots: a user study", The 16th IEEE Int.Symp.on Robot and Human Interactive Communication, Jeju, South Korea, 26-29 Aug. 2007, IEEE, pp. 938-943. http://doi.org/10.1109/ROMAN.2007. 4415218.

Helmer, R., Yassine, A. and Meier, C. (2010), "Systematic module and interface definition using component design structure matrix", Journal of Engineering Design, Vol. 21 No. 6, pp. 647-675. https://doi.org/10.1080 / 09544820802563226.

Höltta, K.M. and Salonen, M.P. (2003), "Comparing three different modularity methods", ASME 2003 Int. Design Engineering Technical Conferences and Computers and Information in Engineering Conference, DETC2003/DTM-48649, Chicago Illinois, pp. 533-541.

Jiao, J.R., Simpson, T.W. and Siddique, Z. (2007), "Product family design and platform-based product development: a state-of-the-art review", Journal of Intelligent Manufacturing, Vol. 18 No. 1, pp. 5-29.

Kahn, P.H., Freier, N.G., Kanda, T., Ishiguro, H., Ruckert, J.H., Severson, R.L. and Kane, S.K. (2008), "Design patterns for sociality in human-robot interaction", The 3rd ACM/IEEE International Conference on Human Robot Interaction, Amsterdam, The Netherlands, ACM, New York, NY, USA, March 12 - 15, 2008, pp. 97-104. http://doi.org/10.1145/1349822.1349836. 
Kory Westlund, J., Lee, J.J., Plummer, L., Faridi, F., Gray, J., Berlin, M., Quintus-Bosz, H., Hartmann, R., Hess, M., Dyer, S. and Dos Santos, K. (2016), "Tega: a social robot”, The 11th ACM/IEEE International Conference on Human Robot Interaction, New Zealand, March 7-10, 2016, IEEE Press, pp. 561-561.

Lee, S.B. and Yoo, S.H. (2017), "Design of the companion robot interaction for supporting major tasks of the elderly", 14th International Conference on Ubiquitous Robots and Ambient Intelligence, Jeju, South Korea, 28 June-1 July 2017, IEEE Press, pp. 655-659. http://doi.org/10.1109/URAI.2017.7992695.

Li, Z., Cheng, Z., Feng, Y. and Yang, J. (2013), “An integrated method for flexible platform modular architecture design”, Journal of Engineering Design, Vol. 24 No. 1, pp. 25-44. https://doi.org/10.1080/09544828.2012.668614.

Li, B.M. and Xie, S.Q. (2015), "Module partition for 3D CAD assembly models: a hierarchical clustering method based on component dependencies", International Journal of Production Research, Vol. 53 No. 17, pp. 5224-5240. https://doi.org/10.1080/00207543.2015.1015748.

Liu, J., Jiang, H., Li, Z. and Hu, H. (2009), “A small window-cleaning robot for domestic use”, International Conference on Artificial Intelligence and Computational Intelligence, Shanghai, China, 7-8 Nov. 2009, IEEE Press, Vol. 2, pp. 262-266. http://doi.org/10.1109/AICI.2009.112.

Lopez, A., Paredes, R., Quiroz, D., Trovato, G. and Cuellar, F. (2017), "Robotman: A security robot for humanrobot interaction", 18th International Conference on Advanced Robotics, Hong Kong, China, 10-12 July 2017, IEEE Press, pp. 7-12. http://doi.org/10.1109/ICAR.2017.8023489.

Okita, S.Y., Ng-Thow-Hing, V. and Sarvadevabhatla, R. (2009), "Learning together: ASIMO developing an interactive learning partnership with children", 18th IEEE International Symposium on Robot and Human Interactive Communication, Toyama, Japan, 27 Sept.-2 Oct. 2009, IEEE Press, pp. 1125-1130. http://doi.org/10.1109/ROMAN.2009.5326135.

Ozer, I. and Erden, Z. (2018), "Literature-based identification of behavioural modules for social robots using design structure matrix", 18th International Conference on Machine Design and Production, Eskisehir, Turkey.

Pahl, G. and Beitz, W. (1996), Engineering Design-A Systematic Approach, The Design Council, London.

Pimmler, T.U. and Eppinger, S.D. (1994), "Integration analysis of product decompositions", ASME 6th International Conference on Design Theory and Methodology, Minneapolis, MN.

Potnuru, A., Jafarzadeh, M. and Tadesse, Y. (2016), “3D printed dancing humanoid robot "Buddy" for homecare", IEEE International Conference on Automation Science and Engineering, Fort Worth, TX, USA, 21-25 Aug. 2016, IEEE Press, pp. 733-738. http://doi.org/10.1109/COASE.2016.7743475.

Qiao, L., Efatmaneshnik, M., Ryan, M. and Shoval, S. (2017), "Product modular analysis with design structure matrix using a hybrid approach based on MDS and clustering”, Journal of Engineering Design, Vol. 28 No. 6, pp. 433-456. https://doi.org/10.1080/09544828.2017.1325858.

Rangan, M.K., Rakesh, S.M., Sandeep, G.S.P. and Suttur, C.S. (2013), “A computer vision based approach for detection of fire and direction control for enhanced operation of fire fighting robot", Int. Conf. on Control, Automation, Robotics and Embedded Systems, Jabalpur, India, 16-18 Dec. 2013, IEEE Press, pp. 1-6. http://doi.org/10.1109/CARE.2013.6733740.

Salichs, M.A., Barber, R., Khamis, A.M., Malfaz, M., Gorostiza, J.F., Pacheco, R., Rivas, R., Corrales, A., Delgado, E. and Garcia, D. (2006), "Maggie: A robotic platform for human-robot social interaction", IEEE Conference on Robotics, Automation and Mechatronics, Bangkok, Thailand, 1-3 June 2006, IEEE Press, pp. 1-7. http://doi.org/10.1109/RAMECH.2006.252754.

Stiehl, W.D., Breazeal, C., Han, K.H., Lieberman, J., Lalla, L., Maymin, A., Salinas, J., Fuentes, D., Toscano, R., Tong, C.H. and Kishore, A. (2006), "The huggable: a therapeutic robotic companion for relational, affective touch", ACM Special Interest Group on Computer Graphics and Interactive Techniques Conference, Boston, MA, USA, July 30 - August 03, 2006, ACM, pp. 15.

Stolarz, J.O. and Rybski, P.E. (2007), An architecture for the rapid development of robot behaviours, Robotics Institute, Carnegie Mellon University, Pittsburgh, PA, USA.

Stone, R.B., Wood, K.L. and Crawford, R.H. (2000), "A heuristic method for identifying modules for product architectures”, Design Studies, Vol. 21 No. 1, pp. 5-31. https://doi.org/10.1016/S0142-694X(99)00003-4.

Téllez, R.A., Ferro, F., Garcia, S., Gomez, E., Jorge, E., Mora, D., Pinyol, D., Poyatos, J., Torres, O., Velazquez, J. and Faconti, D. (2008), "Reem-B: An autonomous lightweight human-size humanoid robot", 8th IEEERAS International Conference on Humanoid Robots, Daejeon, South Korea, 1-3 Dec. 2008, IEEE Press, pp. 462-468. http://doi.org/10.1109/ICHR.2008.4755995.

Xiong, X., Song, Z. and Zhang, J. (2009), "Domestic robots with multi-function and safe internet connectivity", International Conference on Information and Automation, Zhuhai, Macau, China, 22-24 June 2009, IEEE Press, pp. 277-282. http://doi.org/10.1109/ICINFA.2009.5204935.

Zaraki, A., Mazzei, D., Giuliani, M. and De Rossi, D. (2014), "Designing and evaluating a social gaze-control system for a humanoid robot”, IEEE Transactions on Human-Machine Systems, Vol. 44 No. 2, pp. 157-168. http://doi.org/10.1109/THMS.2014.2303083. 